\title{
Design and Evaluation of Aerospace Components for SLM
}

\author{
Roland STOLT ${ }^{1}$, Fredrik ELGH and Tim HEIKKINEN \\ Industrial Product Development, Production and Design, Jönköping University, \\ Sweden
}

\begin{abstract}
Currently, the additive manufacturing process SLM (selective laser melting) is of high interest in the aerospace industry for the manufacture of jet engine components. This is driven by several factors such as reducing weight and minimizing the variation in the manufacturing process. In the paper, the state of practice in designing SLM parts is examined showing that there is plenty of opportunity to adapt designs to the process. However, this is often too time consuming in the early stages. By examining the state of art in SLM part design, the paper and identifies the variant specific cost drives that are proposed to be used to rank the manufacturability of different design alternatives for turbine frame aerospace components.
\end{abstract}

Keywords. SLM, Additive Manufacturing, Aerospace, Design for Additive Manufacturing, DFAM, Manufacturability

\section{Introduction}

For the aerospace industry, the weight of components is of very high importance. Consequently, manufacturers of aircraft components are striving to reduce the weight of their products. For a particular manufacturer of aircraft engines, the interest has turned to the additive manufacturing (AM) method Selective Laser Melting (SLM) hoping to reduce product weight and decrease variation in quality. SLM is a laser powder bed fusion (L-PBF) printing method by which layers of metal powder is laid out and melted together. This makes it possible to print geometrically complex light-weight metal structures. There are currently few printed aircraft turbine components on the market, but examples can be found as for example the fuel injection nozzles of the Leap engine [1]. Siemens has qualified printed vanes for use in power plant turbines in 2018 [2].

SLM does not have many geometric constraints, but there can be big differences in the manufacturing time, cost and quality for an SLM part if it is well adapted to the process or not. Therefore, it is worth to put effort in designing for SLM especially if a series of products are planned. There are plenty of possibilities of designing parts for the SLM process. It involves the minimization of the part weight, orienting the part in the printer, designing support structures to conduct heat and support downfacing surfaces. It also involves determining the printer settings and predicting the expected properties. In addition, designing a part for SLM also involve the planning of the postprocessing and handling, involving a variety of operations [3].

\footnotetext{
${ }^{1}$ Corresponding Author, Email: roland.stolt@ju.se.
} 
However, it is quite time consuming and complicated to get all details perfect. In the early phases of design thorough analysis may be too time consuming. To spend weeks analyzing and optimizing a rough design suggestion that is likely to be changed would probably be a waste of time and resources. To drive the design of new SLM parts towards increased manufacturability, there is a need for a rapid assessment of the manufacturability. A limited number of criteria is needed to determine if one suggested design variant is better or worse from a manufacturability point of view than another. Rapid assessment means that design variants with low manufacturability can be discontinued or reworked. By making the design suggestions more accurate, detailed analyses can be started on higher level design suggestions.

The purpose of this paper is to identify essential manufacturability considerations for SLM manufacture of aerospace parts and propose how to represent them in a model for early stage manufacturability assessment.

The paper contains a state of practice description in AM design from which these essential manufacturability items are identified to be incorporated into an assessment model.

\section{Literature}

To save time and increase the quality of products, all life cycle aspects should be considered already in the early phases of design. This is the general idea in concurrent engineering [4]. To concurrently include production aspects in design is particularly important since producing the part often is a major cost and there are good possibilities of reducing it in the design phase. Consequently, the research fields design for manufacturing (DfM) and design for assembly (DfA) [5] have emerged. In addition to advice and recommendations on how to make the geometry of a part adapted to the intended production process, a way of comparing the manufacturability of different design suggestions is needed. In [6] three key metrics for good producibility is presented namely: quality, time and cost. In their paper, the term producibility is used rather than manufacturability to empathize a holistic approach which is taken in aerospace industry. Manufacturability is a narrower term concentrating on the production process. There are examples of how cost has been used as a measure of manufacturability, such as [7] that introduces a "tolerance cost" for the purpose of comparing different machining processes. Thus, the least costly alternative to obtain the required quality in the least time represents the highest producibility.

In AM, DfA has got its own designation - Design for additive manufacturing (DFAM). This is motivated by that in AM there are possibilities to optimize the product for the production process to a larger extent than in traditional production processes. We are only in the beginning of developing the DFAM paradigm [8]. In the review article [9] there is an interesting overview of the state of art in DFAM encompassing a variety of AM processes. In the field of DFAM, there are publications that propose general recommendations of how to design for the process [10-12]. Orientation in the printer is a key factor for the obtaining good manufacturability as elaborated in [13].

There is also research interest in cost and sustainability of AM processes. In [14], a cost breakdown of AM processes is presented allowing the expected production cost of AM components to be calculated. There are also studies on the packaging of parts in the machine build envelope which has an impact on the per part cost [15]. The cost model can be elaborated further so that the individual component cost for a build with 
components with different geometries can be calculated. This has been proposed by [16]. Their cost model is very refined containing aspects like powder degradation. The model also includes the time for preparing the print, machine related operations like powder change and maintenance. It allows detailed studies of the per part price when stacking several parts on the build plate or reducing component volume by lattice structures.

An aspect of increasing importance is sustainability. In [17] there is an attempt to identify the research gaps in this field in the AM field. Authors recommend more research efforts on energy saving, reduction of waist, occupational health, lifecycle assessment and crosscutting and policy issues.

There are, as mentioned, ample possibilities to optimize products for the AM process compared to traditional production processes. Very complex light weight structures with gradient density can be produced. A commonly used tool for creating these structures is topology optimization (TO). By TO, the material of the part can be distributed the places where it is needed given the load case and boundary conditions. It is can be described as automated computational redesign of the part geometry for a minimized weight, based on finite element analysis. The ideas behind TO is not a new. It has been discussed for a considerable time [18]. However, AM has made it possible to manufacture the geometrically complex structures resulting from TO. In addition, extra leverage for TO in the context of additive manufacturing is obtained by the fact that minimizing the amount of material has a strong positive influence on the manufacturability of the AM part. Less material means not only savings in material but also shortened printing time and less risk of distortion. Consequently, TO is becoming an integral part of DFAM. There is extensive research in TO algorithms that are adapted to better serve the needs of AM design. Examples include considering overhangs [19] and the support structures and build orientation $[20,21]$. There is a high interest in predicting the results of the AM process. Examples include mechanical properties, residual stresses and expected distortions. Process simulation is emerging as an tool in driving DFAM [22]. Quite recently 3-4 years or so, commercial software for the simulation of L-PBF processes have appeared with product available from software vendors.

\section{State of practice in DFAM}

In the design of the AM part, two sets of constraints must be handled. It is the functional requirements that the component must comply with in service, and it is the constraints of the AM process. As with all DfM, it is very useful with a close integration of the two sets to minimize the number of iterations needed. After having come up with a conceptual design for an AM part, several measures are taken to prepare it for manufacturing by AM. An overview of these steps is shown in figure 1.
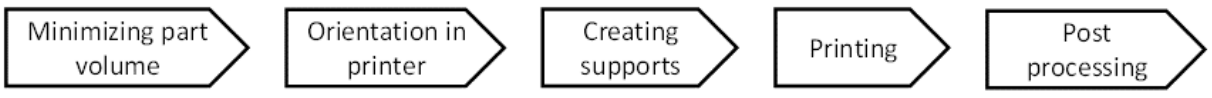

Figure 1. The AM preparation steps.

The part volume needs to be minimized and extensive thickness variation avoided to keep the printing time and risk of geometrical distortion low. In some applications, weight minimization is important from a functional point of view as for example in aerospace and automotive applications. Thus, the weight may already have been minimized in the design phase. If not, preparing for print must start with weight minimization. The weight minimized component must comply with the functional 
requirements of the part, why it is very useful in AM design to always minimize the weight in the design phase even if it is not a functional requirement.

The orientation in the printer include two considerations. Firstly, the build height is kept low since the number of layers that must be laid out directly influence the printing time. Secondly the number and sizes of downfacing surface needs to be minimized. Downfacing surfaces in an angle of less than a certain value (often 30 degrees) to the build plate needs to be supported. This require support material and thereby adding to the manufacturing time and cost.

Geometries that are unsuitable for printing and cannot be alleviated by re-orienting the part have to be improved by local redesign of the geometry. The figure 2 below shows an example of a local improvement. The ending for the round hole to the left has a 0 degree overhang which will result in a poor surface quality. To the right, the shape of the hole has been modified to give a 30-degree angle at the end.

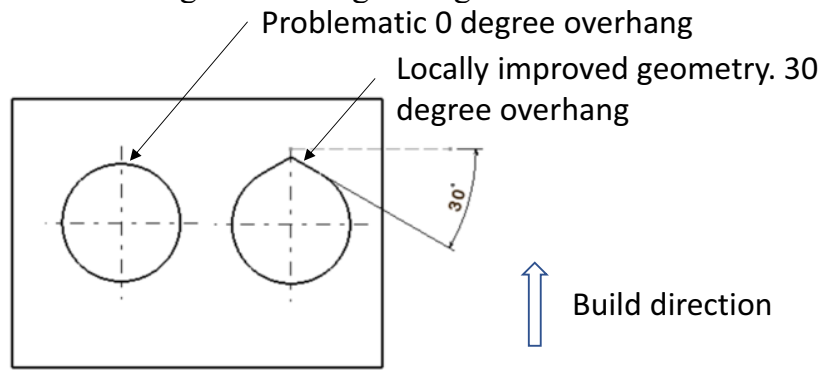

Figure 2. Local geometry modifications

After the part has been oriented, the support structures are created, and the part is printed. The preparation for the print is done in specialized software such as 3DXpert (3dsystems) or Magics (Materialise). With these software, support structures can be generated and the CAD-model is divided into many slices corresponding to layers in the print. A print file is generated that control the operations of the printer. After print, the part is taken out of the printer, and the part is sent to postprocessing which often include machining to remove the support structures and to get the specified surfaces and tolerances.

\subsection{Interaction between the design and the manufacturing domains}

As mentioned, to reduce the number of iterations, there must be a very close interaction between the design and manufacturing domains. The figure 3 illustrates the exchange.

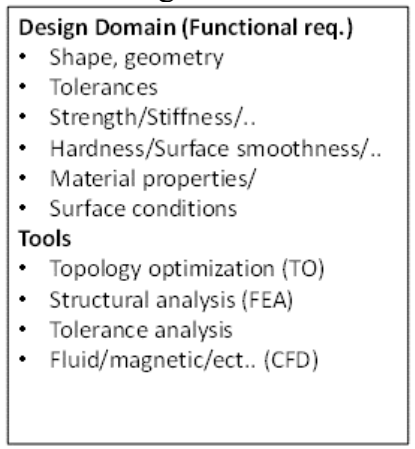

\begin{tabular}{|c|}
\hline $\begin{array}{l}\text { Manufacturing Domain (Manufacturing req.) } \\
\text { - Orientation } \\
\text { - Volume } \\
\text { - Buld height } \\
\text { - Volume of support material } \\
\text { - Expected material properties (directional) } \\
\text { - Expected distortions and stress state. } \\
\text { - Expected time and cost } \\
\text { Tools } \\
\text { - Process simulation } \\
\text { - Print preparation software } \\
\text { - Capp }\end{array}$ \\
\hline
\end{tabular}

Figure 3. Exchange between design and manufacturing domains. 
Firstly, to design for AM require knowledge about the process. The orientation in the printer should be considered already in the early design stages. Efforts should be made to bring down the volume of the part and to minimize the number of low angles downfacing surfaces. As mentioned, TO is often used for minimizing the part volume. Functionality for including constraints like minimum permissible overhangs has started to appear in commercial TO code. The post processing needed to obtain the required surfaces and tolerances should be planned in the design domain. This will perhaps include inserting features for clamping the part in the subsequent machining operations. A preliminary shape is created that can be brought into the manufacturing domain. There, it can be finally oriented, and the support structures can be created using the print preparation software. This makes it possible to use the software to make a prediction of the manufacturing time and cost using a print time estimation and estimation of the amount of powder that will be required for the build. This includes both part and support material.

In SLM, the resulting properties can be expected to be anisotropic and therefore the orientation in the printer cannot be governed only by minimizing the build height and amount of support. Orienting the part to get the best properties where needed may be equally important especially if the part has been optimized to utilize these properties fully. The expected properties can be predicted by process simulation. Process simulation can also be used to predict residual stresses and expected distortions. These can be considered in the design domain to counteract deformation by making geometric compensations. If the residual stresses are not removed by heat treatment, they need to be included when assessing the compliance with requirements in the design domain. By simulating the thermal conduction in the process simulation, the support structures can be modified to redistribute heat and whereby counteracting unwanted phenomena such as deformation or cracking.

To improve the result of the AM process, the printer parameters can be adjusted for the part to be printed. Examples of parameters are the scan speed, laser power and the hatch spacing. It is also possible to defocus the laser to change the size and shape of the melt pool. Also, the order and pattern in which the laser scans are made can be altered. This is referred to as the scan strategy. By planning the scan strategy, material properties can be improved, and negative thermal effects can be counteracted. By adjusting the printing parameters, it is possible to make the resulting part more dense. This has a direct positive effect on the material properties. It is often necessary to print samples with varying printing parameters to understand the effect of changing printing parameters. A DOE can be used. The results of the printing experiments can be used to calibrate the process simulation.

In the design of AM components, there is a complex mutual exchange between the design and manufacturing domains, involving a variety of tools. TO, orientation, support structure design and process simulation can be treated as an integrated multi-objective optimization problem. The results of the evaluations from the manufacturing domain can be used to drive the design towards wanted goals in a optimization loop.

All of this may be necessary when creating highly optimized high end components. However, not all components require such a thorough procedures. For components where the materials properties are not fully utilized, it should for example be possible to not take the expected anisotropy into account, largely simplifying the problem. Likewise, in the early stages of design such thorough analysis may not be feasible. It is then useful to simplify the problem starting from the most basic considerations such as minimizing the amount of material both in the part and support structure. 
It the early stages of design, when different alternatives are considered, there is a need of a "light weight" manufacturability evaluation. This is to check that basic DFAM considerations have been followed and to design in ways that do not add unnecessarily to the manufacturing costs. With such evaluation it will be possible to make a more accurate first design suggestion. Important evaluation points in early stages is the amount of part and support material, and the amount of postprocessing required. Thus, it should be possible to rank the manufacturability of different design suggestions by calculating the expected "cost" for printing and postprocessing. It will not be the cost in absolute terms but rather cost drivers that are variant specific allowing a comparison between diffident design alternatives. This will be elaborated and applied to a component in the next chapter.

\section{Example of design for $A M$}

The component that have been used as a test example in this paper is a turbine frame component. It transfers the forces from the turbine shaft to the aircraft frame. A simplified version of such a component is shown in figure 4 to the left. It is made from 12 sectors seen to right in figure 4 . the. The sectors are then joined together by welding to a complete turbine frame.

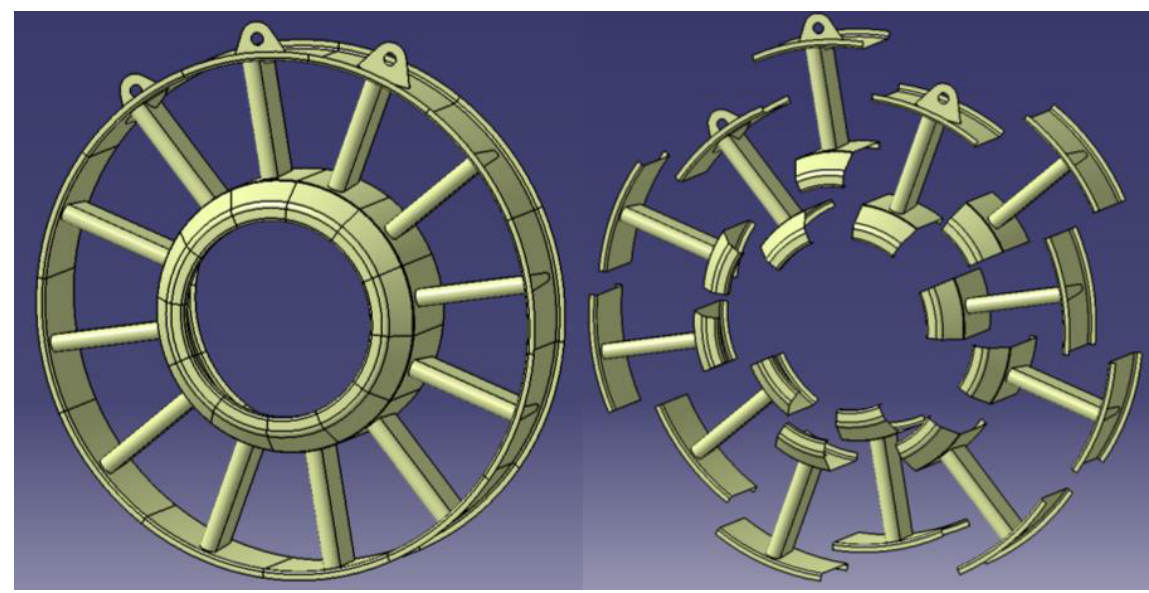

Figure 4. Turbine Frame

The turbine frame is a high end component. It must be manufactured exactly to specification. No defects are allowed. In the early phases of design, it is not possible to make any detailed analysis. It would take too long time and be too costly considering the draft state of the design suggestions Still, different design variants are to be compared with each other to gain knowledge on the response on the manufactirability by changing design parameters. Quick manufacturability analysis is therefore needed. In the case of AM parts, the estimated manufcturing cost is a feasible measure to rank different design proposals.

No special efforts have been made to redesign the sectors for printing. CAD-models of the sectors are imported into a print preparation program (3Dxpert) They are oriented to minimize the overhangs and thereby the amount of support material, see figure 5 . 


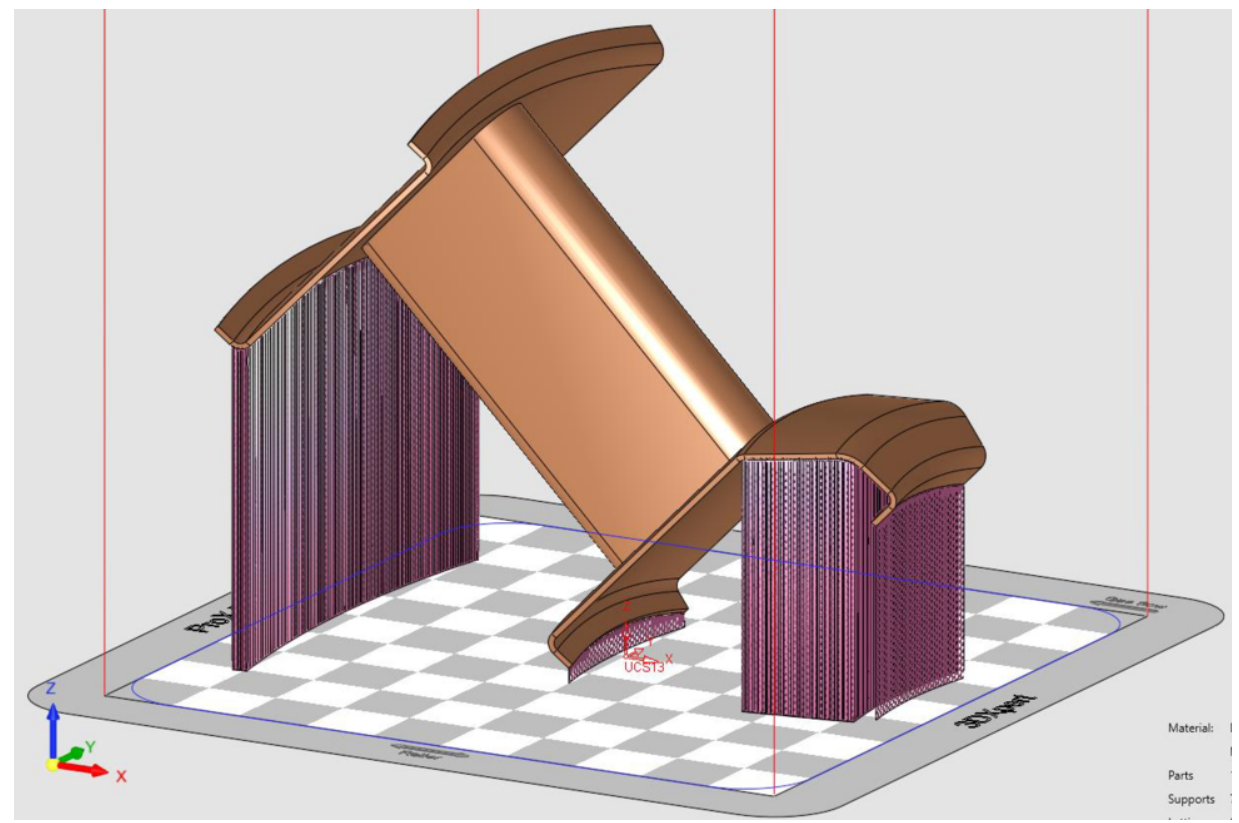

Figure 5. Orientation and support structures.

\subsection{Determining the part cost}

The material and printing parameters are set, and the model is sliced. In the software a printability check can be made to highlight any potential problems such as internal voids or disconnected surface patches. From the preparation program it is possible to get an estimated print time base on the number of layers and the length of the scan path. This time is simply multiplied by the hourly machine cost. The expected amount of powder multiplied by the powder cost is added. It includes both support structures and the part itself. Thus, the cost is calculated by the print preparation software according to:

$$
\operatorname{Cost}_{A M}=C_{m} \cdot \text { time }+C_{p} \cdot \text { mass }
$$

$C_{m}$ - Machine cost [currency/hour]

$$
C_{p^{-}} \text {Cost of powder [currency } / \mathrm{m}^{3} \text { ] }
$$

The machine cost includes all cost for the machine, both value depreciation and consumables like electricity and gas. It is a parameter that the user must enter in the software. However, this is not the total manufacturing cost. There is also pre and postprocessing in several steps. First time is spent preparing the printer and print file, after print, the part must be removed from the printer. This is a manual operation where the non-melted powder in the build volume first is removed. This is often done manually, but automated solutions are starting to appear. An example is SLM ${ }^{\circledR} H U B$ from German company SLM solutions.

When the build has been taken out of the printer, it must be separated from the build plate. This is ideally done by Wire Electrical Discharge Machining (WEDM). Thereafter, 
the part is machined to remove the support structures and to obtain the surfaces and tolerances needed. It should be noted that it is also possible to machine when the parts are still on the build plate. Thereafter, the parts are inspected and then sent for assembly. In the assembly, sectors are welded together to form a complete frame.

Since the cost calculation is only for comparison of different design alternatives, the handling and preparation time can be disregarded based on the assumption that all variants will have the roughly the same time. What remains is the cost of the WEDM separation of the part and the removal of the support structure. The cost of WEDM is directly related to the cross-sectional area of the supports and the cost of machining is related to the volume of the support material.

Thus, the cost of postprocessing can be obtained:

\section{$C_{W E D M^{-}}$Cost of WEDM [currency $\left./ \mathrm{m}^{2}\right]$}

\section{$C_{M i^{-}}$Cost of Milling [currency $/ \mathrm{m}^{3}$ ]}

The complete cost model will then be:

$$
\text { cost }=\text { Cost }_{A M}+C_{W E D M} \cdot \text { Area }_{S M}+C_{M i} \cdot \text { Volume }_{S M}
$$

Where Area $_{\mathrm{SM}}$ is the cross-sectional area of the support material and Volume $\mathrm{SM}_{\mathrm{SM}}$ is the total volume of the support material. Both these quantities are obtained from the print preparation program. With the above expression, different design alternatives can be compared.

\section{Discussion}

In the paper, the interaction between the design and manufacturing domains in SLM part design has been described. It is a complex interaction offering many possibilities of optimizing the part geometry for the process. It is argued that a simplification is needed in the early design stages leading to a suggested cost model with parameters that can be directly obtained from the print preparation software. This allows a ranking of the manufacturability for different design alternatives. Many cost items such as the cost of preparation and part handling is left out under the assumption that it will be the same for all alternatives. The calculations will not give a per part cost in absolute terms. The primary reason is instead gaining knowledge on how manufacturability is affected by design variation. The model only included items related to the printing. The actual case comprises many more manufacturing operations such as machining, assembly, heat and surface treatments, and inspection. To accurately rank the design suggestions these cannot be left out. Similar variant specific calculations must be made and added to the printing cost. Suggestions have been made in [23-25] showing how to make a rapid early stage assessment of the welding cost. There are several other manufacturability related items such as the reachability in welding and in inspection [26]. In all cases the problem is finding criteria that quickly can be evaluated and yet capturing the manufacturability aspects with enough precision to rank different design alternatives in early stages.

It is a matter of identifying the most influential factors and then assessing how difficult and costly these can be expected to be. A cost model combined with "difficulty indices" seems to be a feasible way forward. These indices are easily quantifiable measures of the difficulty in manufacturing. Examples of indices are the number of 
surfaces that cannot be inspected with standard methods combined with the requirement that all surfaces must be inspectable with any known methods. Designs that cannot be inspected must be discontinued or reworked.

It is not yet known how the simple cost model rank the design alternatives. Further is does not include the quality aspect of manufacturability. However, result from the process simulation and actual production data can be used to refine the predictive model considering for example the predicted properties and anisotropies and the expected geometrical deviation adding the quality dimension to the manufacturability evaluation.

\section{Conclusions and future work}

This paper has shown that in SLM part design, there is a very close integration between the design and manufacturing domains. This leads to plenty of opportunity for design optimization based on computational techniques. However, this is in early design stages often too complex and time consuming. A simplification that accurately can rank design suggestions according to their manufacturability is needed. In the paper cost items that are specific to design variants have been identified. It is suggested that a cost can be calculated and used for the ranking of design alternatives according to their manufacturability. In future work the model is planned to be tested on several design variants. This is a step towards automated design evaluation in which a large number of automatically generated design alternatives can be evaluated to build knowledge on how printing manufacturability is affected by the variation.

\section{Acknowledgement}

Participating companies and "Region Jönköpings län" are greatly acknowledged for financing and contributing to this research under the project "ProAct"

\section{References}

[1] Petch, M. GE aviation celebrates 30,000th 3d printed fuel nozzle. 2018 2019-03-11]; Available from: https://3dprintingindustry.com/news/ge-aviation-celebrates-30000th-3d-printed-fuel-nozzle$141165 /$.

[2] Siemens. Additive Manufacturing: Siemens uses innovative technology to produce gas turbines. 2018 [cited 2019 March 11]; Available from: https://www.siemens.com/press/en/feature/2014/corporate/2014-03-3d-druck.php.

[3] I. Bahnini et al., Additive manufacturing technology: the status, applications, and prospects. International Journal of Advanced Manufacturing Technology, 2018. 97(1-4): pp. 147-161.

[4] B. Prasad, Concurrent Engineering Fundamentals. Prentice Hall, Upper Saddle River, 1997.

[5] G. Boothroyd, Product design for manufacture and assembly. 2. ed., rev. and expanded. ed, Dekker, New York, 2002.

[6] J. Vallhagen, , et al., An Approach for Producibility and DFM-methodology in Aerospace Engine Component Development. Procedia CIRP, 2013. 11: p. 151-156.

[7] F. Schultheiss, S. Hägglund and J.E. Ståhl, Modeling the cost of varying surface finish demands during longitudinal turning operations. International Journal of Advanced Manufacturing Technology, 2016. 84(5-8): pp. 1103-1114.

[8] A.W. Gebisa and H.G. Lemu, Design for manufacturing to design for Additive Manufacturing: Analysis of implications for design optimality and product sustainability, Procedia Manufacturing, 2017. 13: p. 724-731. 
[9] M.K. Thompson et al., Design for Additive Manufacturing: Trends, opportunities, considerations, and constraints. CIRP Annals - Manufacturing Technology, 2016. 65(2): p. 737-760.

[10] J.W. Booth, et al., The Design for Additive Manufacturing Worksheet. Journal of Mechanical Design, 2017. 139(10): p. 1-9.

[11] J. Boyer et al., Special Section: Designing for additive manufacturing recent advances in design for additive manufacturing. Journal of Mechanical Design, Transactions of the ASME, 2017. 139(10), 100901.

[12] C. Klahn, B. Leutenecker and M. Meboldt. Design strategies for the process of additive manufacturing. Procedia CIRP. 2015, Vol. 36, pp. 230-235.

[13] B. Leutenecker-Twelsiek, C. Klahn and M. Meboldt. Considering Part Orientation in Design for Additive Manufacturing. Procedia CIRP, 2016, Vol. 50, pp. 408-413.

[14] A. Mahadik, and D. Masel, Implementation of Additive Manufacturing Cost Estimation Tool (AMCET) Using Break-down Approach. Procedia Manufacturing, 2018. 17: p. 70-77.

[15] V. Griffiths et al., Cost-driven build orientation and bin packing of parts in Selective Laser Melting (SLM). European Journal of Operational Research, 2019. 273(1): pp. 334-352.

[16] L. Rickenbacher, A. Spierings and K. Wegener, An integrated cost-model for selective laser melting (SLM). Rapid Prototyping Journal, 2013. 19(3): p. 208-214.

[17] D. Rejeski, F. Zhao, and Y. Huang, Research needs and recommendations on environmental implications of additive manufacturing. Additive Manufacturing, 2018. 19: p. 21-28.

[18] M.P. Bendsøe, Topology optimization : theory, methods and applications. 2. ed., corr. pr.. ed, ed. Springer, Berlin Heidelberg, 2004.

[19] A.T. Gaynor and J.K. Guest, Topology optimization considering overhang constraints: Eliminating sacrificial support material in additive manufacturing through design. Structural and Multidisciplinary Optimization, 2016. 54(5): p. 1157-1172.

[20] Y.H. Kuo et al., Support structure design in additive manufacturing based on topology optimization. Structural and Multidisciplinary Optimization, 2018. 57(1): pp. 183-195.

[21] Langelaar, M., An additive manufacturing filter for topology optimization of print-ready designs. Structural and Multidisciplinary Optimization, 2017. 55(3): p. 871-883.

[22] D.L. Conover, S. Pilz and A.C. To. Simulation-driven product development for additive manufacturing. Simulation for Additive Manufacturing 2017, Sinam 2017. 2017, pp. 97-98.

[23] T. Heikkinen et al., Automated Producibility Assessment Enabling Set-Based Concurrent Engineering, Advances in Transdisciplinary Engineering, Vol. 4, IOS Press, Amsterdam, 2016, pp. 947-956.

[24] D. Kveselys, Weld Producibility Assessment System : Evaluation of Producibility Assessment tools using Set-based approach in Multi-disciplinary Aerospace Design, Master thesis, Jönköping University, 2017.

[25] V.K. Rao Pabolu, R. Stolt and J. Johansson, Flexible manufacturability analysis applied to the welding process to increase sustainability in engineer to order businesses. International Journal of Agile Systems and Management, 2017, 10(3-4): pp. 271-294.

[26] R. Stolt, F. Elgh and P. Andersson, Design for Inspection - Evaluating the Inspectability of Aerospace Components in the Early Stages of Design, Procedia Manufacturing, 2017. 11: p. 11931199. 